\title{
The effect of physical activity on fatigue among survivors of colorectal cancer: a systematic review and meta-analysis
}

\author{
D. Brandenbarg ${ }^{1}$ (I) • J. H. W. M. Korsten ${ }^{1}$ - M. Y. Berger ${ }^{1}$ • A. J. Berendsen ${ }^{1}$
}

Received: 19 May 2017 / Accepted: 2 October 2017 /Published online: 23 October 2017

(C) The Author(s) 2017. This article is an open access publication

\begin{abstract}
Purpose Favorable health outcomes among cancer survivors are increasingly being attributed to lifestyle factors like physical activity, which is now promoted in clinical guidelines. However, the available evidence indicates that physical activity may also reduce fatigue in this patient group. In this systematic review, we aimed to examine whether physical activity could reduce fatigue among survivors of colorectal cancer. Methods The databases of Medline, CINAHL, and PsycINFO were systematically searched, using combinations of MeSH and free-text terms for colorectal cancer, physical activity, and fatigue. Randomized controlled trials and cohort studies with longitudinal data collection were included. We performed a random-effect meta-analysis.

Results Seven studies were included, five were randomized controlled trials, and two were cohort studies. A meta-analysis of the randomized controlled trials, which comprised 630 survivors in total, failed to show that physical activity had a significant effect on fatigue (standardized mean difference $=0.21$ ( -0.07 to 0.49$)$ ); however, reduced levels of fatigue were observed in all studies. The results for the cohort studies were inconclusive: one showed that increasing levels of physical activity were significantly associated with decreasing levels of fatigue; the other showed that decreasing levels of fatigue were not associated with increasing levels of physical activity.
\end{abstract}

D. Brandenbarg

d.brandenbarg@umcg.nl

1 Department of General Practice, University of Groningen, University Medical Center Groningen, P.O. Box 196, 9700

AD Groningen, The Netherlands
Conclusions Based on the data reviewed, we cannot draw definitive conclusions about the effects of physical activity on fatigue. None of the included studies were performed among fatigued survivors of colorectal cancer. More research is needed in this population, ensuring that the trials are appropriately powered to find differences in fatigue.

Keywords Colorectal neoplasms $\cdot$ Fatigue $\cdot$ Exercise $\cdot$ Survivors $\cdot$ Physical activity

\section{Introduction}

Favorable health outcomes among cancer survivors are increasingly being attributed to lifestyle factors, especially to physical activity (PA), which is promoted in clinical guidelines [1]. PA has been shown to improve physical fitness [2] and quality of life [3] and has been suggested to affect mortality and recurrence rates favorably [4]. However, the latter findings are based on observational evidence and need to be interpreted with caution. Moreover, evidence suggests that PA has the potential to reduce fatigue among survivors of cancer [5], though most studies examining the effect of PA on fatigue have been conducted among heterogeneous survivor groups that mainly comprise survivors of breast cancer. Whether PA is equally effective in reducing fatigue among survivors of other cancers, such as colorectal cancer (CRC), has not been thoroughly examined to date. This distinction is important because these patients are generally older and have many disease-specific side effects related to their treatments and co-morbid conditions.

CRC is the third most common cancer worldwide, having age-standardized incidence rates in developed countries of 36.3 and 23.6 per 100,000 for men and women, respectively [6]. Mortality rates, however, are decreasing in these countries 
thanks to improved screening programs, treatments, and follow-up regimes [6-8]. Consequently, the number of survivors of CRC is expected to rise [9], which presents challenges to the provision of follow-up care $[10,11]$. Survivors of CRC show a variety of late and long-term side effects, such as bowel dysfunction [12], anorectal dysfunction, sexual dysfunction, and psychosocial problems [13], which can significantly affect quality of life (QoL) and functioning.

A frequently occurring problem that affects QoL is cancerrelated fatigue [14], which is reported to be twice as prevalent among survivors of CRC compared with an age- and gendermatched reference population [15]. Moreover, it can persist for a prolonged period, being present up to 10 years after diagnosis $[16,17]$. The relationship between PA and fatigue has been studied in cross-sectional studies of CRC survivors, indicating that higher levels of moderate-to-vigorous PA are associated with lower levels of fatigue [18-21]. In other crosssectional research, it was also suggested that fatigue could mediate the relation between PA and QoL [22]. Due to the design of these studies, no conclusions can be made about causality.

In this study, we aimed to conduct a systematic review of the effect of posttreatment PA on fatigue among survivors of $\mathrm{CRC}$, using data from randomized controlled trials (RCTs) or prospective cohort studies.

\section{Methods}

\section{Protocol and registration}

The protocol for this review is available at PROSPERO, the international database of prospectively registered systematic reviews for health and social care (registration number RD42015029702).

\section{Eligibility criteria}

Studies were included if they met the following criteria: they must be a randomized clinical trial, controlled clinical trial, or prospective cohort study; use longitudinal data collection and analysis; report data about patients with CRC; include patients treated with curative intent; use a PA intervention (for the clinical trials) or an assessment of PA (for the cohort studies); assess fatigue using a validated fatigue questionnaire or a fatigue subscale from a validated QoL questionnaire; and have the data published in peer-reviewed journals.

\section{Information sources and search strategy}

The databases of Medline, CINAHL, and PsycINFO were systematically searched in December 2015, using combinations of MeSH and free-text words for "colorectal cancer," "physical activity," and "fatigue." Table 1 shows the full search details for all databases; the search criteria were combined with the "AND" statement. No date restrictions or language restrictions were defined a priori. In addition, we checked the references of included studies for additional publications, and if necessary to obtain the relevant data, we contacted the authors of studies that did not report the results for CRC separately.

\section{Data collection}

Studies were screened for eligibility based on titles and abstracts, after which full-text articles were reviewed, by two researchers independently (DB and JK). Data collection was performed in Microsoft ${ }^{\circledR}$ Access ${ }^{\circledR}$, using a piloted and digitized data extraction form. We identified authors, titles, study types, populations, inclusion and exclusion criteria, intervention details, questionnaires used, and outcomes measured.

\section{Statistical analysis for meta-analysis of randomized controlled trials}

Because of expected heterogeneity of study participants, interventions used, and timing of outcome measurements, we used a random-effects model to pool the effect of PA on fatigue. We calculated standardized mean differences (SMDs) using mean scores and standard deviations at 6 months for the intervention and control groups in all studies. When these data were not available in the original articles, they were calculated by the researchers. When studies varied in time points of measurement of outcomes, we used the SMDs for the time points closest to 6 months. Statistical heterogeneity was evaluated by the $\chi^{2}$ and $I^{2}$ tests and was determined statistically significant for $P<0.1$ and $I^{2}>50 \%$, respectively.

\section{Risk of bias assessment}

Risk of bias assessment was performed using the Cochrane collaboration's risk of bias assessment tool for the controlled trials [23] or the Newcastle-Ottawa quality assessment scale for the cohort studies [24]. Two researchers (DB and JK) independently reviewed the articles. Disagreement was calculated by Cohen's kappa, and discrepancies were discussed until consensus was reached, with arbitration by a third researcher (AJB) when necessary. Publication bias was assessed by evaluating a funnel plot. All analyses were conducted using the computer program Review Manager (version 5.3. Copenhagen, The Cochrane Collaboration, 2014). 
Table 1 Search strategy for different databases

\begin{tabular}{|c|c|c|}
\hline & Medline/Pubmed & CINAHL/PsycINFO \\
\hline Colorectal cancer & $\begin{array}{l}\text { “Colorectal Neoplasms”[Mesh] OR } \\
\text { Colorectal cancer[tiab] OR } \\
\text { colorectal neoplasm*[tiab] OR } \\
\text { colon cancer[tiab] OR rectal } \\
\text { cancer[tiab] OR colon } \\
\text { neoplasm*[tiab] OR rectal } \\
\text { neoplasm*[tiab] OR bowel } \\
\text { cancer[tiab] OR gastrointestinal } \\
\text { cancer[tiab] OR gastrointestinal } \\
\text { neoplasm*[tiab] OR cancer [tiab] }\end{array}$ & $\begin{array}{l}\text { (MH “Colorectal Neoplasms+") OR (MH “Colonic } \\
\text { Neoplasms+") } \\
\text { OR TX ("colorectal cancer") OR TX } \\
\text { (“colon cancer") OR TX (“rectal cancer") }\end{array}$ \\
\hline Physical activity & $\begin{array}{l}\text { "Exercise"[Mesh] OR Exercise*[tiab] } \\
\text { OR physical activit*[tiab] OR } \\
\text { walk*[tiab] OR jog*[tiab] OR } \\
\text { run*[tiab] OR move*[tiab] OR } \\
\text { active lifestyle*[tiab] OR } \\
\text { sport*[tiab] OR lifestyle } \\
\text { intervention*[tiab] OR resistance } \\
\text { training [tiab] OR sedentary } \\
\text { behavior [tiab] }\end{array}$ & 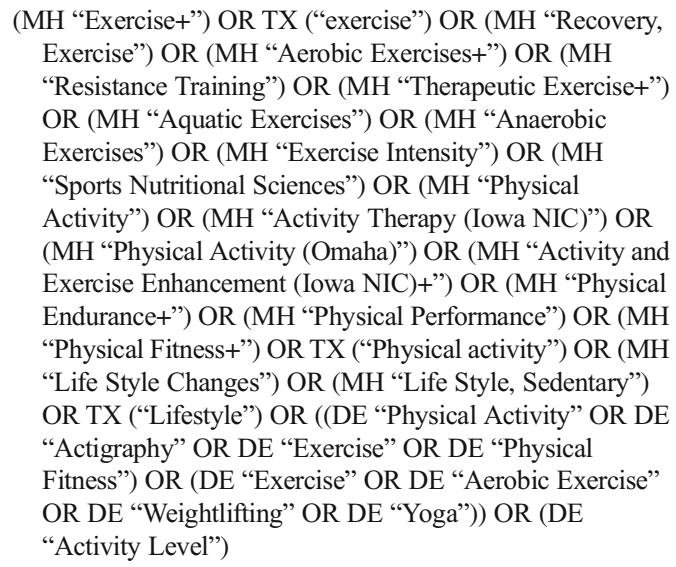 \\
\hline Fatigue & $\begin{array}{l}\text { "Fatigue"[Mesh]) OR } \\
\text { "Questionnaires"[Mesh]) OR } \\
\text { "Quality of Life"[Mesh] OR } \\
\text { Questionnaire*[tiab] OR } \\
\text { HRQOL[tiab] OR fatigue[tiab] OR } \\
\text { quality of life[tiab] OR qol[tiab] OR } \\
\text { daily activit*[tiab] OR } \\
\text { performance[tiab] OR } \\
\text { function*[tiab] OR health related } \\
\text { quality of life [tiab] OR tiredness } \\
\text { [tiab] OR FACT-F OR BFI OR FFIS } \\
\text { OR CFS OR FSI OR MFI OR } \\
\text { MFSI-SF OR EORTS QLQ-C30 } \\
\text { OR CRDFS OR MAF OR FSS OR } \\
\text { FIB }\end{array}$ & $\begin{array}{l}\text { (MH "Fatigue+") OR TX ("Fatigue") OR (MH “Cancer } \\
\text { Fatigue") OR (MH "Mental Fatigue") OR (MH "Quality of } \\
\text { Life+") OR TX ("HRQL") OR TX ("QoL") OR } \\
\text { TX("Quality of Life") OR TX ("Health Related Quality of } \\
\text { Life") or TX ("tiredness") OR TX ("performance") OR (DE } \\
\text { "Fatigue") OR (DE "Quality of Life" OR DE "Quality of } \\
\text { Work Life") }\end{array}$ \\
\hline
\end{tabular}

Search strings for each part were combined using the "AND" Boolean statement

\section{Results}

\section{Selection of articles}

Figure 1 summarizes the search and article selection process. In total, we found 1196 records and screened the titles and abstracts of 1118 records after removing duplicates; agreement between researchers was moderate (Cohen's kappa of 0.558). After removing unsuitable articles, we screened 61 full texts (Cohen's kappa was 0.693, which was considered good), which led to the exclusion of another 54 records. Therefore, 7 records were included for data extraction: 5 were RCTs (one of which was a pilot), and 2 were cohort studies in which data were analyzed prospectively [25-31].

\section{Randomized controlled trials}

\section{Patients}

Table 2 shows the characteristics of the five included RCTs. In total, 630 CRC survivors were included, with sample sizes ranging from 18 to 410 patients. The average ages of survivors ranged from 57.3 to 69 years, most patients were male, and all patients were included within 5 years after treatment. In all studies but that by Pinto et al., patients with metastatic cancer were excluded [28].

The baseline characteristics of the intervention and control groups are summarized in Table 3. Mean ages ranged from $59.5(11.2)$ to $68.7(9.13)$ years in the intervention groups, and 
Fig. 1 Flow diagram showing the path of article selection

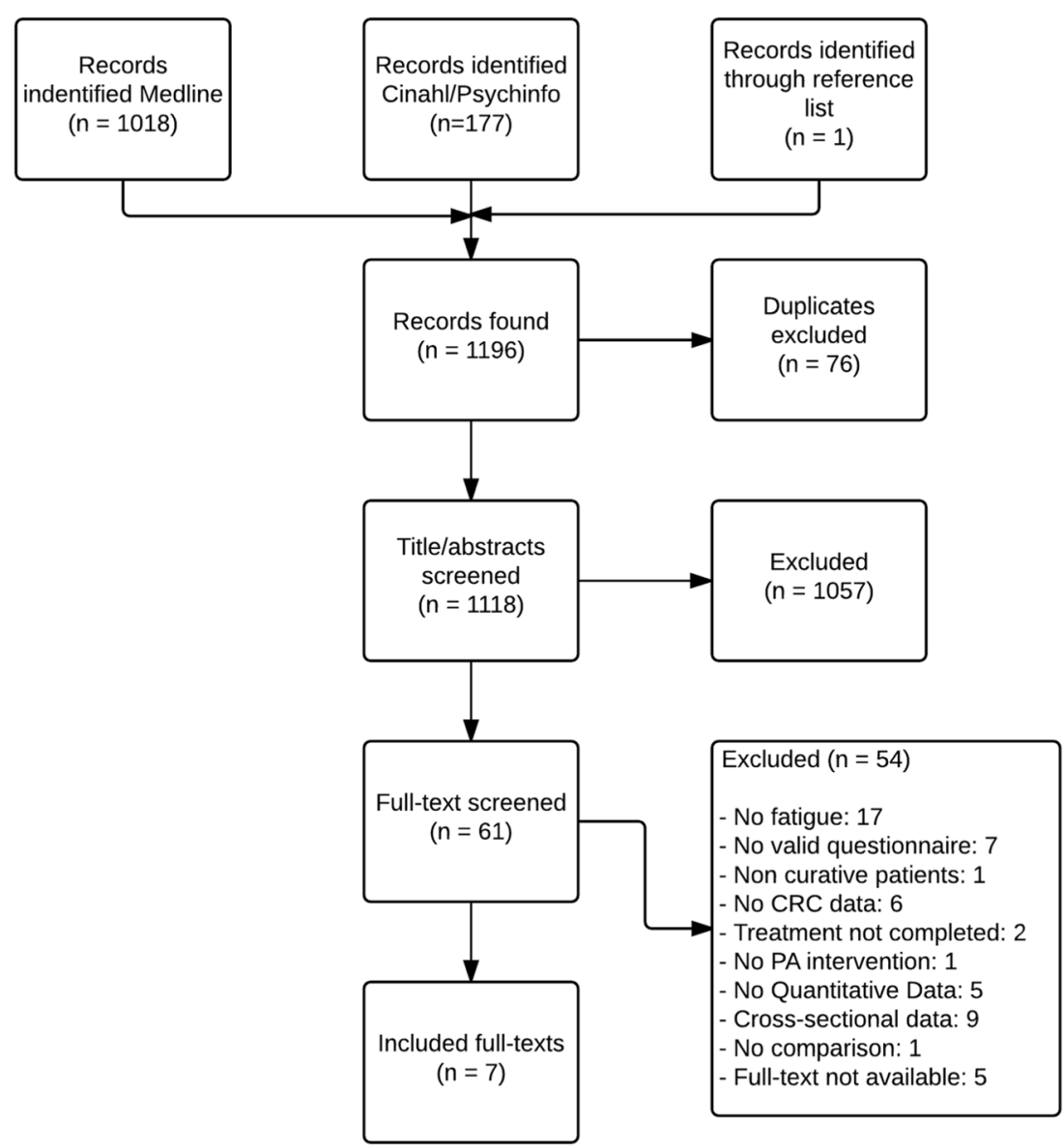

from $55.6(8.24)$ to $70.3(8.7)$ years in the control groups. In three of five studies [25-27], patients in the intervention groups were younger than those in the control groups. Four of five studies [25-28] included more women in the intervention group (44-60\%) compared to the control group (22.2$54 \%)$.

\section{Physical activity interventions}

Interventions in the included RCTs (Table 2) ranged from 10 weeks to 36 months, with a median duration of 13 weeks. Three interventions were home-based, requiring participants to exercise themselves, and focused on either moderate cardiovascular activity for up to $30 \mathrm{~min} 3-5$ times per week [25, 28 ] or encouraging participants to increase their annual number of steps walked to 10,000 [27]. To enhance adherence, the home-based programs used monitoring or counseling telephone calls. The other two interventions consisted of supervised exercise sessions combined with advice for participants to exercise in their own time $[26,30]$. One intervention involved a combination of cardiovascular exercise and resistance training [26], whereas the other consisted of traditional hatha yoga [30]. In most of the studies, controls received care as usual, but, in one case, this was supplemented by weekly calls to monitor symptoms and a survivorship tip sheet [28]. The study by Cramer et al. used patients on a waiting list as their control population [30]. Dropout rates in included interventions ranged from 6 to $21 \%$, with the larger studies having larger dropout rates.

\section{Outcome measures}

The primary outcome measures are detailed in Table 2. All RCTs used the Functional Assessment of Cancer TherapyFatigue (FACT-F) questionnaire to measure fatigue as a secondary outcome measure. We contacted the corresponding author of one study with unusual low fatigue scores, to find out that the scores had been recoded [25]. We transformed these scores back to the original scale and used them for the meta-analysis.

The fatigue outcomes for the RCTs are summarized in Table 3. All studies showed improvements in fatigue scores from baseline to after the intervention. However, the effect of the intervention on fatigue, when compared with the control group, was only statistically significant in one of the studies [26].

Figure 2 shows the results of the random-effects meta-analysis of the effect of the PA interventions on fatigue, which 


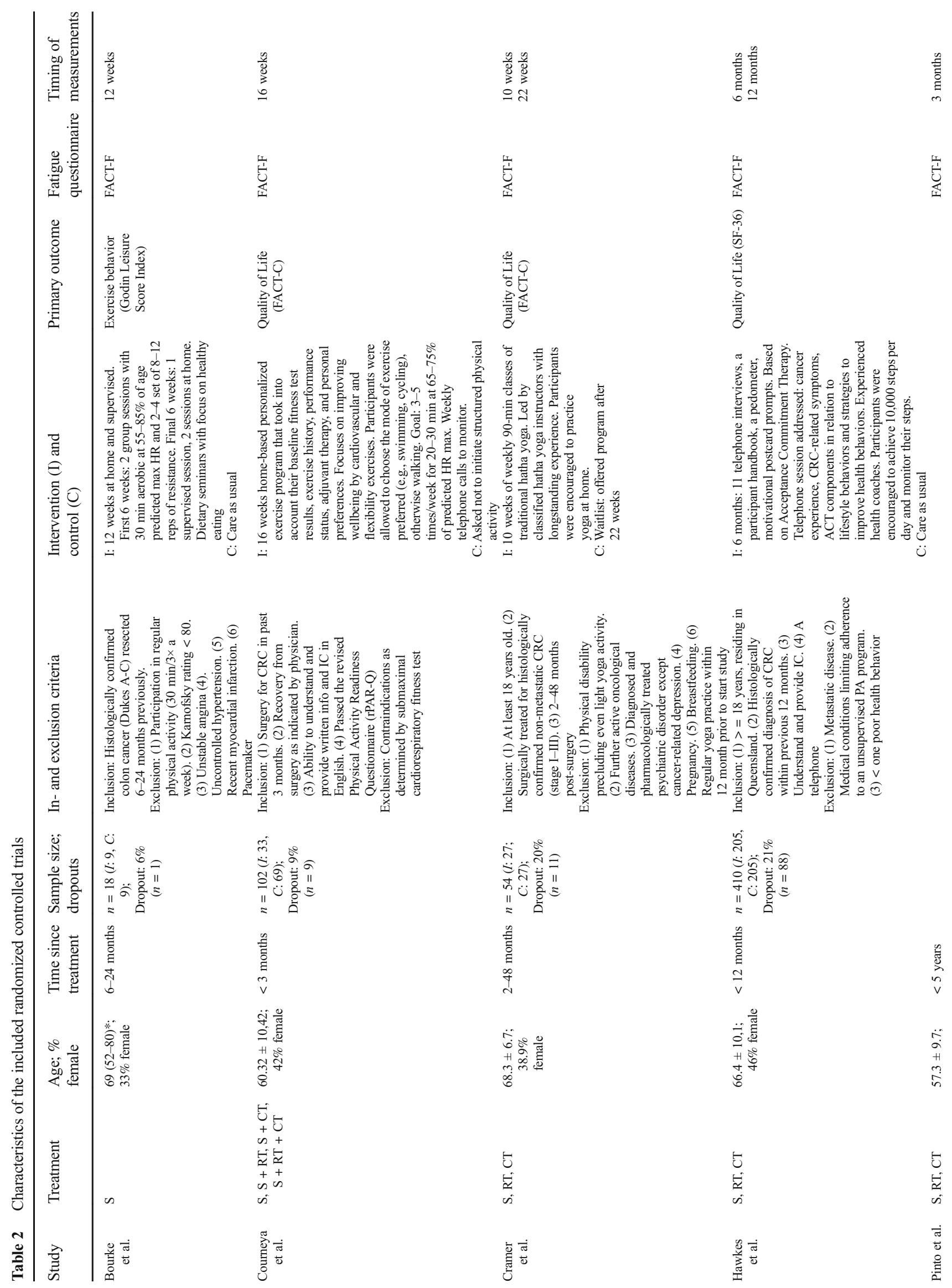


confirmed that there was no significant effect on fatigue in patients with treated CRC $(\mathrm{SMD}=0.21(-0.07$ to 0.49$)$, $\left.I^{2}=42 \%, Z=1.46, p=0.14\right)$. No statistically significant heterogeneity was found between the studies $\left(\chi^{2}=6.95\right.$ $\left.(p=0.14), I^{2}=42 \%\right)$.

\section{Risk of bias}

Figure 3 depicts the risk of bias assessment for each included RCT. Because participant and personnel blinding was not possible in these studies, blinding was evaluated based on that of the outcome assessors. The studies by Hawkes et al. and Cramer et al. were considered to have the lowest risks of bias $[27,30]$, whereas the studies by Courneya et al. and Pinto et al. had the highest risks of bias [25, 28]. These latter two studies also scored high on other bias assessments. For example, contamination (i.e., the control group also increased their levels of PA) was an issue in the study by Courneya et al. [25], and failure to obtain the required numbers based on the sample size calculation was an issue in the study by Pinto et al. [28]. As can be inferred from the funnel plot in Fig. 4, publication bias was unlikely.

\section{Cohort studies}

\section{Patients}

The two longitudinal cohort studies comprised 3904 patients with CRC, and longitudinal data was available in 2828 of these $[29,31]$. The mean age of patients was $69.1 \pm 9.42$ years, $44.4 \%$ were female, and the average time since diagnosis was $5.2 \pm 2.8$ years. Most had non-metastatic CRC.

\section{Outcomes}

Both cohort studies measured fatigue using the Fatigue Assessment Scale. The cohort study by van Putten et al. ( $n=2451$ ) examined the correlation between PA and symptoms or function-related barriers (including fatigue), clinical or sociodemographic variables [31]. While levels of moderate-to-vigorous PA were lower for fatigued CRC survivors compared with those who were not, changes in fatigue scores among survivors were not significantly associated with changes in PA levels $(\beta$ : $-0.25,95 \% \mathrm{CI}:-0.50$ to 0.01 , $p=0.05)$. The other cohort study by Husson et al. $(n=1453)$ analyzed the course of fatigue and its correlates [29]. However, this study did show that increased levels of moderate-to-vigorous PA among CRC survivors were significantly associated with decreased levels of cancer-related fatigue over time $(\beta$ : $-0.03,95 \% \mathrm{CI}:-0.05$ to $-0.01, p<0.01)$. 


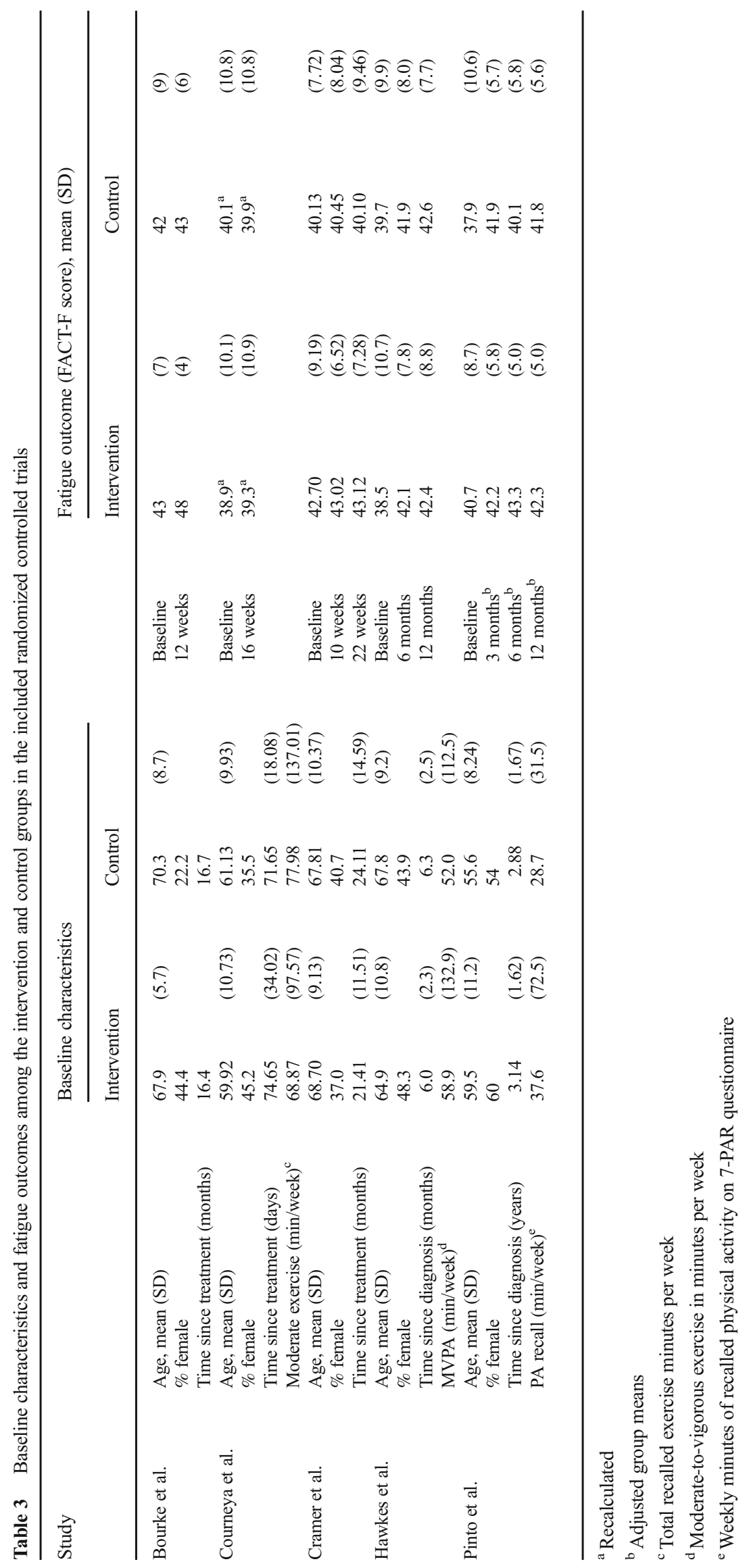




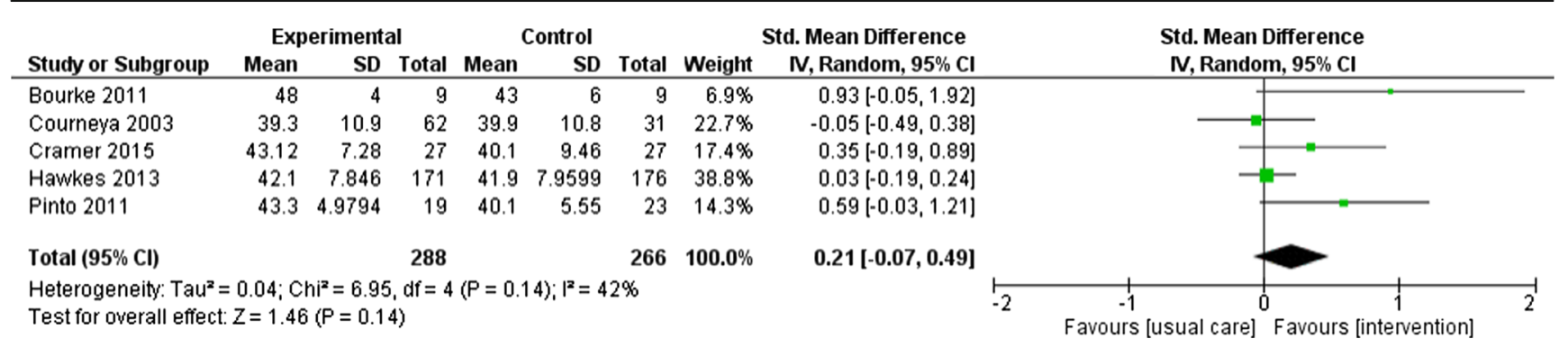

Fig. 2 Forest plot for the meta-analysis of the randomized controlled trial results

\section{Quality assessment}

The studies were performed using comparable methodology; therefore, the quality assessments were comparable. In both studies, participants were somewhat representative of the average CRC survivors in their communities (1 star), and the exposed and non-exposed cohorts were also enrolled from the same communities (1 star). Ascertainment of exposure (fatigue or moderate-to-vigorous PA) was based on written self-reporting (no star), as were the outcome assessments (no star), and the outcome of interest could have been present at the start of the study (no star). Both studies controlled for most important factors (1 star) and had a sufficiently long follow-up for the outcome to occur (1 star). Both studies suffered from substantial loss to follow-up (approximately 60\%), thereby receiving no star for this criterion. Therefore, the studies scored four out of eight possible stars.

\section{Discussion}

\section{Summary}

In this systematic review, we performed a meta-analysis of five RCTs comprising 630 survivors of CRC. Interventions based on increasing PA were shown not to have any significant effect on fatigue levels. However, despite the lack of statistical significance, a trend for reduced fatigue was observed in all intervention groups. We considered the combined effect not clinically relevant. Concerning the longitudinal cohort studies, one showed that increased levels of moderate-tovigorous PA were significantly associated with decreased fatigue, but another showed that decreasing levels of fatigue were not associated with increasing levels of PA.

\section{Comparison with the literature}

Our meta-analysis is the first to focus solely on the effects of PA interventions on fatigue after treatment for CRC. Findings from meta-analyses that included survivors of all types of cancer showed that interventions to increase PA had significant effects on fatigue [3, 32-34], as did a meta-analysis of supervised exercise interventions among breast cancer survivors [35]. However, most of the meta-analyses were over-represented by breast cancer survivors, and they showed considerable heterogeneity. A subgroup analysis in one metaanalysis indicated that the results did not differ by type of cancer [3], though this comparison was only between survivors of breast cancer and survivors of other cancers, without further specification of cancer type.

PA has been shown to be effective in reducing fatigue among people who are healthy or have long-term conditions $[36,37]$. It has been hypothesized that PA can positively affect key biological variables associated with fatigue in cancer survivors, including loss of skeletal muscle mass and strength, anemia, increased proinflammatory cytokines levels, and psycho-behavioral problems [38]. There is no reason to assume that these should be radically different in survivors of CRC.

A possible explanation for not finding an effect in this meta-analysis is that none of the included trials was performed among fatigued survivors. Average fatigue scores at baseline ranged from 38.5 to 43 , yet it is recommended that a score less than 34 be used for the diagnosis of fatigue when using the FACT-F [39]. Given that the scores observed in the included studies were close to average fatigue scores in a normative population (43.6 \pm 9.4) [40], this may have led to ceiling effects in the analyses. This was observed in earlier metaanalyses [32]. Despite randomization protocols, the studies in our meta-analysis (except the studies by Courneya et al. [25] and Hawkes et al. [27]) showed higher FACT-F scores in the intervention groups that could have led to even larger ceiling effects.

In previous meta-analyses, the populations tended to be younger compared with those in the current review. For example, in the meta-analysis by Mishra et al. [3], the average age varied ranged from 39 to 70 years, whereas it ranged from 60 to 69 in the current meta-analysis. It has been suggested that older survivors of cancer benefit less from PA interventions [41, 42], which is possibly related to lower adherence among the elderly [43]. Further research is needed into the optimal types of interventions designed to increase PA among older survivors of cancer [41, 44].

Three of the five studies in our meta-analysis used homebased exercise protocols with telephone monitoring, focusing on aerobic exercise $[25,27,28]$, while a fourth studied the 


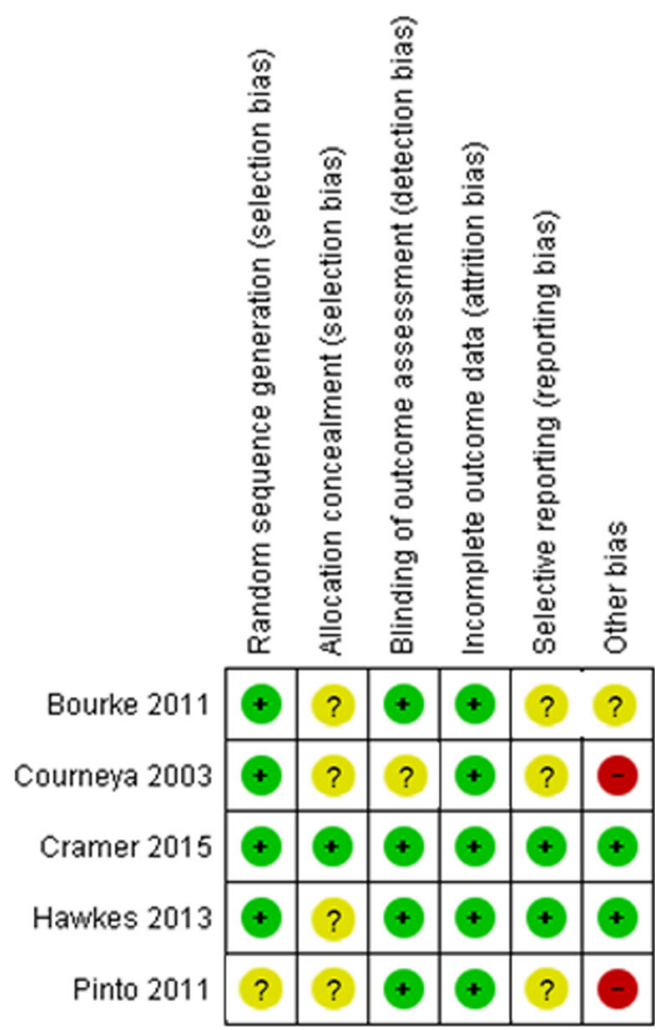

Fig. 3 Risk of bias assessment of the randomized controlled trials

effect of hatha yoga [30]. Earlier research suggested that the effects of PA on fatigue were larger when the intensity of PA was higher [34], and these four studies arguably did not include exercise of sufficient intensity. One trial that combined aerobic and strength exercises, possibly leading to a higher intensity, did show a significant effect. However, this was only a pilot study and was not powered to assess the effects on fatigue, indicating that the results need to be interpreted with caution. It has been suggested that lower intensity intervention might only offer beneficial effects if the exercise routines become a part of daily life [34]. Considering the short duration of most trials in our current meta-analysis, it is questionable whether this could be assessed.

The results from the included cohort studies showed that fatigue levels decreased when survivors of CRC increased their moderate-to-vigorous PA. However, the other showed that PA did not significantly increase when fatigue levels decreased. Thus, PA could positively affect fatigue, but it is likely that other clinical or demographic variables have confounding effects. Although causality cannot be inferred from observational research, selection bias was less of a problem compared with the RCTs. This is particularly relevant because the RCTs of the type we included were more likely to appeal to survivors who were already interested or participating in exercise, and because earlier research has shown that current exercise status is the best predictor for adherence to exercise interventions [45]. The opposite is true of fatigued survivors, those who are not interested in PA, and those who feel more ill, because such people may be less likely to accept an invitation to participate in trials that require intense PA.

\section{Strengths and weaknesses}

A major strength of our review and meta-analysis is that all the included RCTs used the FACT-F questionnaire as the outcome measure when assessing fatigue. The FACT-F has been shown to be sensitive to changes over time [46, 47]. Indeed, although earlier meta-analyses showed large heterogeneity in fatigue outcomes, this was probably related to the differences in the questionnaires used to evaluate fatigue [32, 34]. Also, we did not limit the review to RCTs, which is important because these are especially prone to selection bias in studies of PA, as discussed. Other risks in trials of PA include selective attrition and the potential for contamination, with the possibility that
Fig. 4 Funnel plot for publication bias

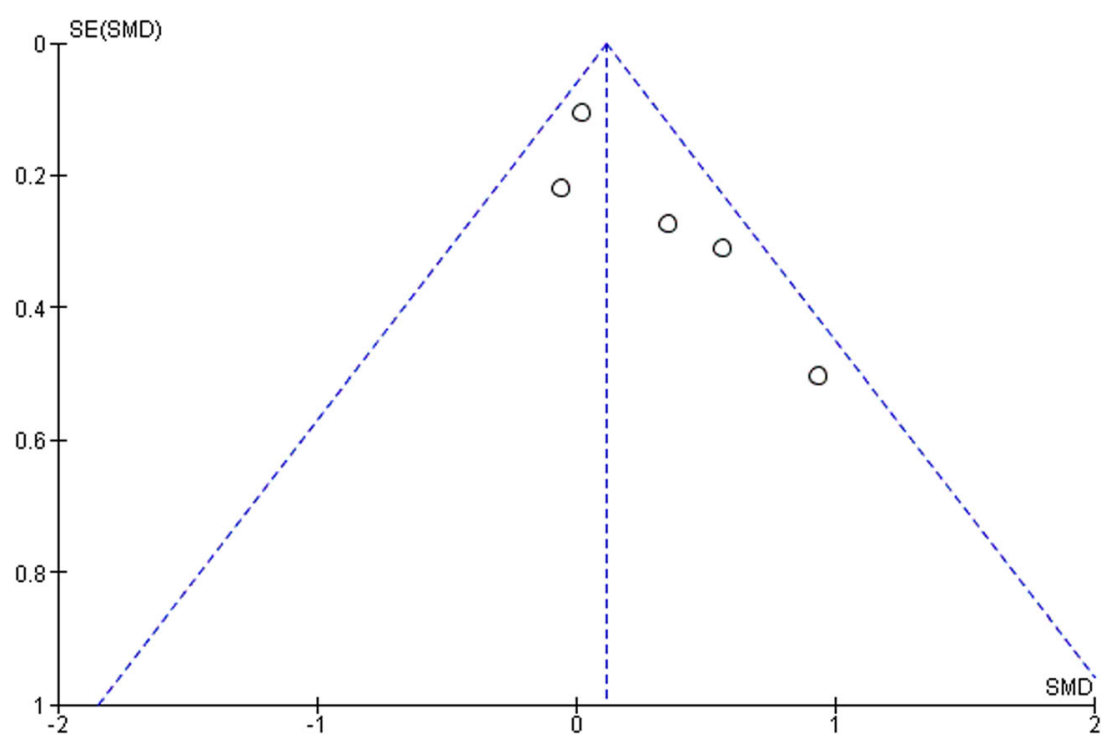


participants randomized to the control group could improve their PA, leading to further underestimation of the effect of the intervention.

Heterogeneity was classified as moderate in our metaanalysis $\left(I^{2}=41 \%\right)$, possibly because of the differences in the types and intensities of PA, or because of the differences in the timings of the outcome measures. We analyzed outcomes that were measured between 6 weeks and 6 months after the intervention, but earlier research has shown that the effects of PA on fatigue varied between these time points. For example, improvements have been seen at 12 weeks and between 3 and 6 months after intervention, but not at 6 months [3]. Another noticeable weakness of our study was the small number of included studies, making it impossible to perform subgroup analyses to compare the effects of either aerobic and strength exercises or self-guided and supervised exercise.

\section{Conclusion}

The results of this systematic review and meta-analysis do not allow for definitive conclusions about the effect of PA on fatigue among survivors of CRC. More research is needed and should be conducted in trials powered to find differences in the reduction of fatigue and that are performed among fatigued survivors of CRC. However, considering the many other beneficial effects of PA on fitness, QoL, and survival, physicians should not hesitate to encourage survivors of CRC to engage in more PA.

Acknowledgements Dr. Robert Sykes (www.doctored.org.uk) provided technical editing and writing services for the final drafts of this manuscript.

\section{Compliance with ethical standards}

Conflicts of interest The authors declare that they have no conflict of interest.

Open Access This article is distributed under the terms of the Creative Commons Attribution-NonCommercial 4.0 International License (http:// creativecommons.org/licenses/by-nc/4.0/), which permits any noncommercial use, distribution, and reproduction in any medium, provided you give appropriate credit to the original author(s) and the source, provide a link to the Creative Commons license, and indicate if changes were made.

\section{References}

1. Rock CL, Doyle C, Demark-Wahnefried W, Meyerhardt J, Courneya KS, Schwartz AL, Bandera EV, Hamilton KK, Grant B, McCullough M, Byers T, Gansler T (2012) Nutrition and physical activity guidelines for cancer survivors. CA Cancer J Clin 62: 243-274
2. Jones LW, Liang Y, Pituskin EN, Battaglini CL, Scott JM, Hornsby WE, Haykowsky M (2011) Effect of exercise training on peak oxygen consumption in patients with cancer: a meta-analysis. Oncologist 16:112-120

3. Mishra SI, Scherer RW, Snyder C, Geigle P, Gotay C (2014) Are exercise programs effective for improving health-related quality of life among cancer survivors? A systematic review and meta-analysis. Oncol Nurs Forum 41:E326-E342

4. Friedenreich CM, Neilson HK, Farris MS, Courneya KS (2016) Physical activity and cancer outcomes: a precision medicine approach. Clin Cancer Res 22:4766-4775

5. Cramp F, Byron-Daniel J (2012) Exercise for the management of cancer-related fatigue in adults. Cochrane Database Syst Rev 11: CD006145

6. Torre LA, Bray F, Siegel RL, Ferlay J, Lortet-Tieulent J, Jemal A (2015) Global cancer statistics, 2012. CA Cancer J Clin 65:87-108

7. Edwards BK, Ward E, Kohler BA, Eheman C, Zauber AG, Anderson RN, Jemal A, Schymura MJ, Lansdorp-Vogelaar I, Seeff LC, van Ballegooijen M, Goede SL, Ries LA (2010) Annual report to the nation on the status of cancer, 1975-2006, featuring colorectal cancer trends and impact of interventions (risk factors, screening, and treatment) to reduce future rates. Cancer 116:544-573

8. Bosetti C, Levi F, Rosato V, Bertuccio P, Lucchini F, Negri E, La Vecchia C (2011) Recent trends in colorectal cancer mortality in Europe. Int J Cancer 129:180-191

9. DeSantis CE, Lin CC, Mariotto AB, Siegel RL, Stein KD, Kramer JL, Alteri R, Robbins AS, Jemal A (2014) Cancer treatment and survivorship statistics, 2014. CA Cancer J Clin 64:252-271

10. McCabe MS, Bhatia S, Oeffinger KC, Reaman GH, Tyne C, Wollins DS, Hudson MM (2013) American Society of Clinical Oncology statement: achieving high-quality cancer survivorship care. J Clin Oncol 31:631-640

11. El-Shami K, Oeffinger KC, Erb NL, Willis A, Bretsch JK, PrattChapman ML, Cannady RS, Wong SL, Rose J, Barbour AL, Stein KD, Sharpe KB, Brooks DD, Cowens-Alvarado RL (2015) American Cancer Society Colorectal Cancer Survivorship Care Guidelines. CA Cancer J Clin 65:428-455

12. Emmertsen KJ, Laurberg S, Rectal Cancer Function Study Group (2013) Impact of bowel dysfunction on quality of life after sphincter-preserving resection for rectal cancer. Br J Surg 100: 1377-1387

13. Birgisson H, Pahlman L, Gunnarsson U, Glimelius B (2007) Late adverse effects of radiation therapy for rectal cancer-a systematic overview. Acta Oncol 46:504-516

14. Hofman M, Ryan JL, Figueroa-Moseley CD, Jean-Pierre P, Morrow GR (2007) Cancer-related fatigue: the scale of the problem. Oncologist 12(Suppl 1):4-10

15. Thong MS, Mols F, Wang XS, Lemmens VE, Smilde TJ, van de Poll-Franse LV (2013) Quantifying fatigue in (long-term) colorectal cancer survivors: a study from the population-based patient reported outcomes following initial treatment and long term evaluation of survivorship registry. Eur J Cancer 49:1957-1966

16. Jansen L, Herrmann A, Stegmaier C, Singer S, Brenner H, Arndt V (2011) Health-related quality of life during the 10 years after diagnosis of colorectal cancer: a population-based study. J Clin Oncol 29:3263-3269

17. Arndt V, Merx H, Stegmaier C, Ziegler H, Brenner H (2004) Quality of life in patients with colorectal cancer 1 year after diagnosis compared with the general population: a population-based study. J Clin Oncol 22:4829-4836

18. Peddle CJ, Au HJ, Courneya KS (2008) Associations between exercise, quality of life, and fatigue in colorectal cancer survivors. Dis Colon Rectum 51:1242-1248 
19. Grimmett C, Bridgewater J, Steptoe A, Wardle J (2011) Lifestyle and quality of life in colorectal cancer survivors. Qual Life Res 20: $1237-1245$

20. Vallance JK, Boyle T, Courneya KS, Lynch BM (2014) Associations of objectively assessed physical activity and sedentary time with health-related quality of life among colon cancer survivors. Cancer 120:2919-2926

21. Van Roekel EH, Bours MJ, Breedveld-Peters JJ, Meijer K, Kant I, Van Den Brandt PA, Sanduleanu S, Beets GL, Weijenberg MP (2015) Light physical activity is associated with quality of life after colorectal cancer. Med Sci Sports Exerc 47:2493-2503

22. Buffart LM, Thong MS, Schep G, Chinapaw MJ, Brug J, van de Poll-Franse LV (2012) Self-reported physical activity: its correlates and relationship with health-related quality of life in a large cohort of colorectal cancer survivors. PLoS One 7:e36164

23. Higgins JPT, Altman DG, Gøtzsche PC, Jüni P, Moher D, Oxman AD, Savović J, Schulz KF, Weeks L, Sterne JAC (2011) The Cochrane Collaboration's tool for assessing risk of bias in randomised trials. BMJ 343:d5928. https://doi.org/10.1136/bmj. d5928

24. Wells G, Shea B, O'Connell D, Peterson J, Welch V, Losos M, Tugwell P (2013) The Newcastle-Ottawa Scale (NOS) for assessing the quality of nonrandomised studies in meta-analyses. http://www. ohri.ca/programs/clinical_epidemiology/oxford.asp. Accessed 12/ 162016

25. Courneya KS, Friedenreich CM, Quinney HA, Fields AL, Jones LW, Fairey AS (2003) A randomized trial of exercise and quality of life in colorectal cancer survivors. Eur J Cancer Care (Engl) 12: 347-357

26. Bourke L, Thompson G, Gibson DJ, Daley A, Crank H, Adam I, Shorthouse A, Saxton J (2011) Pragmatic lifestyle intervention in patients recovering from colon cancer: a randomized controlled pilot study. Arch Phys Med Rehabil 92:749-755

27. Hawkes AL, Chambers SK, Pakenham KI, Patrao TA, Baade PD, Lynch BM, Aitken JF, Meng X, Courneya KS (2013) Effects of a telephone-delivered multiple health behavior change intervention (CanChange) on health and behavioral outcomes in survivors of colorectal cancer: a randomized controlled trial. J Clin Oncol 31: 2313-2321

28. Pinto BM, Papandonatos GD, Goldstein MG, Marcus BH, Farrell N (2013) Home-based physical activity intervention for colorectal cancer survivors. Psychooncology 22:54-64

29. Husson O, Mols F, van de Poll-Franse LV, Thong MS (2015) The course of fatigue and its correlates in colorectal cancer survivors: a prospective cohort study of the PROFILES registry. Support Care Cancer 23:3361-3371

30. Cramer H, Pokhrel B, Fester C, Meier B, Gass F, Lauche R, Eggleston B, Walz M, Michalsen A, Kunz R, Dobos G, Langhorst J (2016) A randomized controlled bicenter trial of yoga for patients with colorectal cancer. Psychooncology 25:412-420

31. van Putten M, Husson O, Mols F, Luyer MD, van de Poll-Franse LV, Ezendam NP (2016) Correlates of physical activity among colorectal cancer survivors: results from the longitudinal populationbased profiles registry. Support Care Cancer 24:573-583

32. Speck RM, Courneya KS, Masse LC, Duval S, Schmitz KH (2010) An update of controlled physical activity trials in cancer survivors: a systematic review and meta-analysis. J Cancer Surviv 4:87-100
33. Puetz TW, Herring MP (2012) Differential effects of exercise on cancer-related fatigue during and following treatment: a meta-analysis. Am J Prev Med 43:e1-24

34. Fong DY, Ho JW, Hui BP, Lee AM, Macfarlane DJ, Leung SS, Cerin E, Chan WY, Leung IP, Lam SH, Taylor AJ, Cheng KK (2012) Physical activity for cancer survivors: meta-analysis of randomised controlled trials. BMJ 344:e70

35. Meneses-Echavez JF, Gonzalez-Jimenez E, Ramirez-Velez R (2015) Effects of supervised exercise on cancer-related fatigue in breast cancer survivors: a systematic review and meta-analysis. BMC Cancer 15:77-015 -1069-4

36. Puetz TW (2006) Physical activity and feelings of energy and fatigue: epidemiological evidence. Sports Med 36:767-780

37. Mock V, Frangakis C, Davidson NE, Ropka ME, Pickett M, Poniatowski B, Stewart KJ, Cameron L, Zawacki K, Podewils LJ, Cohen G, McCorkle R (2005) Exercise manages fatigue during breast cancer treatment: a randomized controlled trial. Psychooncology 14:464-477

38. Al-Majid S, Gray DP (2009) A biobehavioral model for the study of exercise interventions in cancer-related fatigue. Biol Res Nurs 10: 381-391

39. Van Belle S, Paridaens R, Evers G, Kerger J, Bron D, Foubert J, Ponnet G, Vander Steichel D, Heremans C, Rosillon D (2005) Comparison of proposed diagnostic criteria with FACT-F and VAS for cancer-related fatigue: proposal for use as a screening tool. Support Care Cancer 13:246-254

40. Cella D, Lai JS, Chang CH, Peterman A, Slavin M (2002) Fatigue in cancer patients compared with fatigue in the general United States population. Cancer 94:528-538

41. Luctkar-Flude M, Groll D, Woodend K, Tranmer J (2009) Fatigue and physical activity in older patients with cancer: a six-month follow-up study. Oncol Nurs Forum 36:194-202

42. Courneya KS, Friedenreich CM, Sela RA, Quinney HA, Rhodes RE, Handman M (2003) The group psychotherapy and home-based physical exercise (group-hope) trial in cancer survivors: physical fitness and quality of life outcomes. Psychooncology 12:357-374

43. Courneya KS, Segal RJ, Reid RD, Jones LW, Malone SC, Venner PM, Parliament MB, Scott CG, Quinney HA, Wells GA (2004) Three independent factors predicted adherence in a randomized controlled trial of resistance exercise training among prostate cancer survivors. J Clin Epidemiol 57:571-579

44. Rao AV, Cohen HJ (2008) Fatigue in older cancer patients: etiology, assessment, and treatment. Semin Oncol 35:633-642

45. Courneya KS, Friedenreich CM, Quinney HA, Fields AL, Jones LW, Fairey AS (2004) Predictors of adherence and contamination in a randomized trial of exercise in colorectal cancer survivors. Psychooncology 13:857-866

46. Yellen SB, Cella DF, Webster K, Blendowski C, Kaplan E (1997) Measuring fatigue and other anemia-related symptoms with the Functional Assessment of Cancer Therapy (FACT) measurement system. J Pain Symptom Manag 13:63-74

47. Cella D, Eton DT, Lai JS, Peterman AH, Merkel DE (2002) Combining anchor and distribution-based methods to derive minimal clinically important differences on the Functional Assessment of Cancer Therapy (FACT) anemia and fatigue scales. J Pain Symptom Manag 24:547-561 